\title{
Appartenance générationnelle et figures professionnelles
}

\author{
Le cas des artistes de cirque en France
}

\begin{abstract}
Émilie Salaméro', Marine Cordier ${ }^{2}$
[Résumé] L'avènement du nouveau cirque en France dans les années 1970 a inauguré son entrée dans le domaine de la culture subventionnée sur le modèle de la «création ", en même temps que de profondes transformations en matière de socialisation des artistes. Les décennies suivantes ont vu se déployer les processus concomitants d'institutionnalisation, de professionnalisation et de légitimation de ce secteur culturel, ainsi que la scolarisation de l'apprentissage du métier. Cet article montre comment ces changements de contexte ont modifié le recrutement social des artistes de cirque et donné lieu à des figures différenciées en termes de dispositions et de rapport au métier. À partir d'entretiens menés auprès d'une trentaine d'artistes de cirque nés entre 1952 et 1981, l'approche générationnelle proposée ici permet de caractériser deux de ces figures. D'un côté, les artistes du nouveau cirque, polyvalents et soucieux de proposer un art du cirque pour tous, ont accédé au métier grâce à une socialisation affinitaire et informelle tardive, adossée au secteur de l'animation socioculturelle. De l'autre, les artistes de cirque contemporain, sportifs et plus diplômés que les premiers, ont appris leur métier en écoles professionnelles, conduisant à une spécialisation disciplinaire et à mener une carrière dans un paysage professionnel plus institutionnalisé. Issues de deux générations distinctes, ces figures sont associées aujourd'hui à des segments professionnels hiérarchisés.
\end{abstract}

Mots-clés : artistes de cirque ; socialisation ; parcours ; professionnalisation ; génération.

[Abstract] The coming of the new circus in France in the 1970s inaugurated its entrance in the field of subsidized culture on the model of the "creation", alongside profound transformations regarding artists' socialization. During the next decades concomitant processes of institutionalization, professionalization, and legitimization of this cultural sector, as well as the schooling of the job learning deployed. This article shows how these contextual changes modified the social recruitment of circus artists and how differentiated figures emerged in terms of capacities and work perception. From interviews led with around thirty circus artists born between 1952 and 1981, a generational approach allows to characterize two of theses figures. On one side, new circus artists, polyvalent and concerned with proposing a circus art for all, reached the job thanks to a late, informal and peer socialization, leaning on the sector of the social and cultural activities. On the other hand, contemporary circus artists, sportspersons

\footnotetext{
${ }^{1}$ Groupe de recherches sociologiques sur les sociétés contemporaines (EA 3815), Université de Poitiers.

${ }^{2}$ Institutions et dynamiques historiques de l'économie et de la société (UMR 8533), Université Paris Nanterre.
} 
and more qualified than the first ones, learned their trade in professional schools, leading to a disciplinary specialization and to a career conducted in a more institutionalized professional landscape. Coming from two different generations, these figures are associated with hierarchized professional segments.

Keywords: circus artists; socialization; career; professionalization; generation.

En France, le cirque a connu de profonds changements dès les années 1970, période où s'amorcent sa reconnaissance en tant qu'art ainsi que la transformation des modes d'exercice du métier, toutes deux liées à l'arrivée de nouveaux entrants. Jusque-là, la profession reposait principalement sur des entreprises commerciales qui proposaient un spectacle perçu comme un divertissement pour tous : Pinder, Bouglione ou Gruss demeurent les représentants les plus connus du cirque classique français. Les « enfants de la balle » apprenaient le métier au sein de leur famille ou auprès de "maîtres » : socialisations familiale et professionnelle apparaissaient étroitement liées (BarréMeinzer, 2004).

Le contexte post-68 voit émerger un "nouveau cirque» porté par des artistes qui réinventent cette pratique culturelle en se référant au modèle de la "création » artistique. À partir de 1979, l'intervention renforcée du ministère de la Culture, qui fait suite aux difficultés économiques de la profession, contribue aussi à modifier ses contours. Après l'ouverture des premières écoles privées ${ }^{3}$, le ministère encourage le renouvellement de ce groupe professionnel. La création de l’École nationale supérieure des arts du cirque (ENSAC) en 1985 puis la labellisation d'écoles dites " préparatoires » contribuent à imposer la forme scolaire (Vincent, Lahire, Thin, 1994) d'apprentissage du métier ainsi qu'un parcours de multiformation (Menger, 1997). La reconnaissance artistique progressive du cirque ainsi que la transformation de ses modes d'accès favorisent la multiplication des aspirants au métier et leur renouvellement, notamment en ce qui concerne leurs origines sociales et leurs parcours scolaires. Dans les années 2000, ces nouvelles générations sont d'ailleurs désignées par le label « cirque contemporain »(Guy, 2001). En parallèle, la hausse des subventions allouées au secteur contribue à l'essor rapide du nombre de compagnies de cirque évoluant sur un marché plus concurrentiel et plus institutionnalisé qu'auparavant.

Les contextes politiques et institutionnels agissent sur les trajectoires individuelles (Faure, 2004), c'est pourquoi ils constituent ici un axe d'analyse central des parcours (Demazière, Samuel, 2010) d'artistes de cirque, interrogés dans le cadre d'une recherche sur les transitions professionnelles ${ }^{4}$. Les entretiens menés auprès de 33 artistes de

\footnotetext{
${ }^{3}$ En 1974, l'École nationale du cirque dirigée par A. Fratellini et P. Etaix et l'École au Carré, créée par S. Montfort et A. Gruss.

${ }^{4}$ Les entretiens auprès d'artistes de cirque ont été réalisés entre 2014 et 2016 dans plusieurs régions françaises (Ile de France, Occitanie, Nouvelle Aquitaine principalement) dans le cadre d'un programme de recherche "Sorties de scène » (dirigé par S. Julhe et É. Salaméro et soutenu par l'Agence nationale de la recherche), et d'une thèse de doctorat (Cordier, 2009).
} 
cirque âgés de 34 à 64 ans mettent au jour l'existence de générations et de figures professionnelles distinctes, liées aux processus imbriqués d'institutionnalisation, de légitimation et de professionnalisation (Brandl, 2006) qu'a connus le cirque depuis une quarantaine d'années. Si certaines recherches ont souligné le rôle de l'origine sociale dans le rapport au travail des artistes intermittents du spectacle (Sinigaglia, 2013), les effets de l'appartenance générationnelle sur les manières d'exercer et de penser ce métier sont peu explorés. En France, à la suite des travaux analysant les ruptures esthétiques engendrées par l'arrivée de nouveaux entrants (Bourdieu, 1998), il a été montré comment les artistes de nouveau cirque ont cherché à se distinguer de ceux du cirque classique (Cordier, 2007), tant sur les plans esthétique (création d'un spectacle original versus numéros indépendants les uns des autres), éthique (égalité versus hiérarchie entre les membres) et économique (modèle de la compagnie versus société commerciale). Une approche générationnelle permet toutefois de mieux cerner les spécificités des processus de socialisation professionnelle en termes de temps et d'espace (Fournier, 2008). La génération désigne ici des individus nés sur une période donnée, aux profils proches et qui partagent des expériences socialisatrices communes (Demazière, 2004 ; Hammou, 2011) ${ }^{5}$. Depuis le renouvellement du cirque dans les années 1970, plusieurs générations d'artistes de cirque se sont en effet succédées. Bien qu'elles se référent toutes au modèle de la création artistique, elles donnent lieu à des figures professionnelles contrastées en termes de recrutement social, de parcours scolaire et de socialisations artistique et/ou sportive. L'ensemble de ces facteurs façonne les pratiques professionnelles et les rapports au cirque des artistes rencontrés.

L'analyse se concentrera plus particulièrement sur deux générations ${ }^{6}$ qui seront successivement présentées. Nous montrerons en premier lieu comment les artistes de nouveau cirque ont accédé au métier par des voies diverses, notamment celle de l'animation socioculturelle. Puis nous insisterons sur le rôle clé joué par la socialisation sportive et par l'école professionnelle pour les artistes de cirque contemporain.

\section{Artistes de nouveau cirque : une socialisation affinitaire et informelle}

Une première génération est représentée par neuf artistes de notre échantillon, dont trois femmes. Nés entre 1952 et 1968, ils ont fait leurs débuts dans le cirque entre la fin

\footnotetext{
L'échantillon d'artistes interrogés a été constitué sur la base du travail de suivi réalisé par des écoles professionnelles (ENSAC et Lido) et par sollicitation de proche en proche. Les enquêtés ont été pseudonimysés.

${ }^{5}$ Si l'approche générationnelle permet d'articuler histoires individuelle, locale et globale, seules les deux premières échelles sont prises en compte dans cet article.

${ }^{6}$ Nous n'abordons pas dans le cadre de cet article le cas des artistes nés entre 1968 et 1975, qui se situent dans un entre deux vis-à-vis des deux générations choisies pour l'analyse. Ces dernières se présentent comme les plus contrastées en termes de modes de socialisation.
} 
des années 1970 et le début des années 1990. Bien qu'ils aient emprunté des voies diverses pour accéder au métier, ces artistes ont en commun d'avoir commencé cette pratique de manière tardive, la plupart après l'âge de vingt ans. À l'exception d'une acrobate pour qui devenir artiste de cirque représentait un "rêve » d'enfant, pour les autres, le choix de ce métier n'est pas vécu sur le modèle de la vocation. La découverte de cette pratique passe par une activité de loisir initiée en dehors du cercle familial ou d'une école professionnelle, comme en témoigne cet extrait d'entretien :

Bah c'est vraiment par hasard parce que... En fait j'ai, j'ai arrêté grosso modo mes études à dix-huit ans, en ratant brillamment mon bac[calauréat]. [...] Et donc j'avais croisé un mec qu'avait acheté un monocycle et des balles de jonglage en Allemagne, parce qu'en France on n'en trouvait pas. Donc j'ai essayé, puis ça mavait plu quoi. [...] Jean et Sabine étaient déjà depuis quelques mois à cet atelier du Lido. Et donc on s'est mis à jongler tous les trois. Sans but de spectacle, hein. C'était comme ça, pour s'amuser. (Dominique, né en 1957, père artisan électricien, mère au foyer)

D’abord pensée comme un simple passe-temps, la pratique du jonglage ou d'autres disciplines du cirque suscite un investissement grandissant, ce qui permet à certains de convertir ce loisir en une activité professionnelle, notamment dans le secteur de l'animation.

\subsection{De l'animation socioculturelle au monde du spectacle}

Quatre artistes de notre échantillon se sont engagés dans la pratique du cirque grâce à l'école du Lido. Relevant des services socioculturels de la ville de Toulouse, celle-ci comptait parmi les rares espaces de pratique amateur existant dans ce domaine dans les années 1980. Dominique y prend des cours de jonglage alors qu'il est animateur dans une Maison des jeunes et de la culture (MJC), après avoir exercé différents emplois suite à son échec au baccalauréat. Il y rencontre Jean, qui jongle également, en parallèle de son emploi dans une imprimerie, ainsi que Sabine qui avait arrêté ses études dans l'année précédant le baccalauréat et quitté sa famille. Nouant des affinités autour de cette pratique, ils s'entraînent, progressent ensemble et décident finalement de créer une compagnie. Dominique quitte alors son emploi à la MJC tandis que Sabine renonce à passer un diplôme du secteur de l'animation.

De son côté, Pascal a appris sur le tas les bases du jonglage, en parallèle de son emploi de jardinier dans une petite ville. C'est dans une convention de jonglerie qu'il rencontre, à lâge de 21 ans, le directeur du Lido. Celui-ci, qui peine à trouver des enseignants pour son école, lui propose de donner des cours d'initiation au cirque auprès d'enfants. Enthousiaste à cette idée, Pascal démissionne de son poste de fonctionnaire municipal, ne se voyant pas exercer toute sa vie ce métier perçu comme subalterne. «Mon élément déclencheur ça a été que j’allais être papa. [...] Je vais être papa, je ne vais pas me retrouver, je ne vais pas faire jardinier 40 ans ! » (Pascal, né en 1968, père technicien, mère au foyer). Il se forme alors à d'autres techniques de cirque 
et fonde avec deux autres animateurs du Lido une compagnie qui rencontrera un large succès. Son parcours, comme ceux de Dominique et de Sabine, révèle comment l'animation socioculturelle a pu fonctionner pour certains comme une voie de passage vers le métier d'artiste de cirque. En effet, en tant que «domaine d'activité professionnelle lié à des loisirs personnels (théâtre, musique, danse, etc.) »(Lebon, 2013 : 67), l'animation leur a permis de convertir leur pratique amateur du cirque en un emploi rémunéré. Le caractère ouvert du recrutement social des animateurs a en outre facilité l'accès de jeunes peu diplômés et sans formation spécifique à ce groupe professionnel, avant leur passage vers l'exercice d'un métier artistique, synonyme à leurs yeux de mobilité ascendante, car il leur permet d'échapper à des emplois peu qualifiés.

Si la plupart des artistes de cette génération n’ont pas connu de socialisation artistique antérieure au cirque, deux d'entre eux y sont toutefois venus par le biais d'une expérience théâtrale. C'est le cas de Michel, qui a découvert le théâtre au lycée. Issu d'une famille ouvrière de l'Est de la France, il entame des études de physique en Institut universitaire de technologie après son baccalauréat. Au cours de son parcours scolaire et des évènements de Mai 68 qui le marquent lorsqu'il est adolescent, il développe un rejet de la condition salariale et des aspirations à un style de vie « bohème populaire» (Mauger, Poliak, 2008) à l'instar de certaines fractions militantes de la jeunesse populaire. Après avoir vécu des " expériences communautaires » au début des années 1970, il intègre une compagnie de théâtre de rue7. Cette expérience le conforte dans sa volonté de privilégier une forme d'expression capable de susciter l'intérêt d'un public « populaire».

On fait les saltimbanques et ça marche vachement bien, le rapport au public est vachement intéressant. Et avec cette espèce d'idée qu'il faut être dans la rue, aller chercher le public populaire. [...] Et puis je pense que toute forme institutionnelle m'était interdite, par une sorte d'inhibition sociale, comme un interdit d'accéder à un monde qui n'était pas celui des ouvriers, on appellerait ça une trahison de classe quoi. [...] Et le cirque se prêtait à réinventer euh... un monde, puisqu'il est un univers totalement - en tout cas à ce moment-là - en marge. (Michel, né en 1953, père ouvrier dans l'automobile, mère au foyer)

Davantage que ses conventions esthétiques, c'est d'abord l'imaginaire lié au cirque, associé à une certaine marginalité, qui répond aux aspirations de Michel. S'identifier à la figure du « saltimbanque » l'autorise à embrasser une carrière artistique tout en restant à distance d'un monde théâtral perçu comme «bourgeois » et peu accessible aux enfants d'ouvriers. Au début des années 1980, ces représentations l'amènent à fonder, avec d'autres jeunes artistes, une compagnie de nouveau cirque qui est toujours en activité.

${ }^{7}$ Visant à faire de la rue un espace d'expression artistique propice à attirer des publics habituellement «éloignés de la culture » (Gaber, $2014: 72$ ), cette nouvelle forme esthétique traduit une contestation des hiérarchies culturelles et une critique des formes artistiques institutionnalisées, dont le théâtre (Dapporto, Sagot-Duvauroux, 2000). 
Des aspirations similaires se retrouvent chez Patrice, pourtant issu des classes supérieures dotées en capital culturel. Initié au théâtre au lycée, il suspend ses études de médecine pour devenir comédien avant de se tourner vers l'art du clown, forme d'expression corporelle jugée plus universelle. «Pour nous, le théâtre, c'était le corps, le moins de mots possible et puis une vie marginale. Donc le cirque était... nous attendait. [...] Parce qu'on voulait faire un théâtre populaire, itinérant, sans frontière, si possible pas bloqué par la parole, corporel » (Patrice, comédien et clown né en 1952, père directeur de chambre de commerce et d'industrie, mère institutrice). Le duo clownesque fondé par Patrice et sa compagne lui permettra de se produire pendant plus de quinze ans auprès d'un large public en France comme à l'étranger.

Si devenir artiste de cirque est présenté par les enquêtés comme un choix assumé, leurs parents ont eu des difficultés à l'accepter, d'autant plus lorsqu'il impliquait de mettre un terme à des études supérieures et de renoncer ainsi à une voie d'ascension sociale jugée plus sûre.

\subsection{Des réticences familiales à une orientation vers le cirque}

Comme Patrice, trois autres artistes se sont en effet tournés vers le cirque alors qu'ils s'étaient engagés dans des études supérieures, sans nourrir de projet professionnel précis. Développant une pratique circassienne en amateur à travers des stages suivis en parallèle de leur cursus, ils y ont pris goût au point de mettre un terme à leurs études pour se consacrer à l'activité artistique. C'est le cas de Pierre qui, après avoir pratiqué la gymnastique à haut niveau, s'est inscrit en licence de sciences et techniques des activités physiques et sportives (STAPS). Il envisage de passer le concours de professeur d'éducation physique et sportive lorsqu'il est sollicité par le Lido pour y donner des cours aux côtés de Pascal. Il s'éprend alors du cirque, où il peut mobiliser ses dispositions sportives et son goût pour la pédagogie, et qui lui apparaît plus « ouvert » que le monde sportif qu'il fréquentait:

J'hésitais dans mes études aussi. Et donc c'est très bien tombé parce que justement, c'est peut-être une ouverture que je cherchais, et qui m'a ouvert un autre monde au niveau des activités physiques. Pour moi ça l'ouvrait vraiment avec tout le milieu artistique. [...] Par rapport à mon tempérament, j'avais pas besoin de sécurité, au contraire, je m'ennuie vite dans des structures un peu trop fermées. (Pierre, né en 1965, père électricien, mère institutrice)

Craignant de se retrouver "enfermé " dans un emploi de fonctionnaire de l'Éducation nationale et par le caractère rigide du monde sportif, Pierre renonce à son projet initial. Après quelques années passées au Lido comme animateur, il fonde une compagnie avec Pascal. Voyant ses parents surpris par ce choix, Pierre préfère leur dire qu'il travaille comme "professeur dans une école de cirque" plutôt que comme " saltimbanque ", terme qui serait pour eux synonyme de déclassement. Deux autres artistes ont également mis fin à des études universitaires (en mathématiques et en philosophie) pour se tourner vers le cirque. Les parents de Julie regrettent eux aussi son 
choix d'arrêter sa licence de mathématiques. « Peut-être qu'ils s'étaient dit que leur fille serait professeur, et là, quand j'ai fait le clown... Bon maintenant je m'en rends compte, dire "ma fille elle est clown", c'est genre... c'est pas très reluisant quoi. [...] Bon voilà après le tout c'est de rencontrer des choses qui te passionnent» (Julie, née en 1967, père maçon, mère au foyer). Pour plusieurs membres de cette génération, cette orientation professionnelle a ainsi suscité une certaine incompréhension de la part de familles peu familières du monde du spectacle vivant ; être artiste de cirque ne représentait pas, à leurs yeux, un « vrai» métier.

En dépit des réticences exprimées par leur entourage et de l'incertitude inhérente aux carrières artistiques, ces individus ont privilégié une activité vécue sur le mode de la passion. Les artistes du spectacle dans leur ensemble valorisent davantage les gratifications symboliques (épanouissement, autonomie) que la sécurité de l'emploi et les rétributions matérielles (Sinigaglia, 2013). Le faible degré d'institutionnalisation du nouveau cirque et l'absence de barrières formelles à l'entrée (telles qu'une formation professionnelle spécifique) ont pu inciter certains jeunes à se lancer sur cette voie, a priori plus accessible que d'autres (celle du théâtre notamment). Pour les artistes issus de milieux modestes, le cirque a ainsi pu apparaître comme « un instrument raisonnable de mobilité sociale et d'affranchissement du salariat », à l'image du rôle joué par la bande dessinée pour certains dessinateurs (Boltanski, 1975 : 39).

\subsection{Des carrières en marge du marché subventionné de l'art}

Dans un contexte où l'offre de formation professionnelle était encore limitée, la plupart des artistes de cette génération ont appris les techniques de cirque " sur le tas » en s'entraînant entre amateurs, comme le décrit Sabine :

J'avais été à des festivals... Je voyais des gens qui jonglaient, je m'étais dit "ah tiens", enfin ça m'avait plu et du coup j'avais juste euh... je crois que j'avais dû acheter trois balles et puis les massues je m'en étais fabriqué trois, un peu artisanalement [rires]. [...] On essayait [avec Jean et Dominique] de se voir quasi tous les jours, de s'entraîner. (Sabine, née en 1967, père ingénieur, mère secrétaire)

À l'instar des formes d'« autodidaxie collective » observées chez les musiciens de jazz (Coulangeon, 1999), certains savoir-faire tels que la jonglerie ou l'art clownesque (privilégiés par la moitié des artistes interrogés de cette génération) peuvent faire l'objet d'une transmission informelle au sein d'un collectif favorisant imitation et émulation entre pairs. Pour des spécialités nécessitant encadrement et infrastructures, le recours à des cours privés ou des stages devient en revanche nécessaire. Même si des compétences sportives peuvent faciliter l'acquisition de certaines techniques, comme chez un pratiquant d'escalade devenu acrobate aérien, elles ne constituent pas un prérequis indispensable à l'entrée dans le métier, contrairement à la génération suivante. La faible socialisation artistique préalable des artistes de nouveau cirque interrogés n'est, pour la plupart, pas compensée par une socialisation sportive. Cette 
dernière paraît d'autant moins nécessaire que les conventions esthétiques promues par le nouveau cirque font primer l'originalité, la recherche de singularité et la créativité sur la virtuosité technique et la prise de risque (Fourmaux, 2006):

Après y a le truc de faire, d'être dans la création [...]. Moi je jonglais, Luc aussi un peu, on faisait des massues ensemble, Marie dansait, enfin ça allait pas très loin : le grand écart et puis on faisait de la musique, y avait des éléments un peu clownesques aussi, mais l'univers était là, c'était le plus important. (Michel, né en 1953, père ouvrier dans l'automobile, mère au foyer)

La découverte tardive du cirque et l'apprentissage du métier sur le tas n'ont pas empêché les artistes de cette génération d'intégrer rapidement un marché du travail artistique en pleine expansion à partir des années 1980 (David-Gibert, 2006), dynamisé par les demandes d'animation de la part de collectivités locales et de petites structures culturelles. Le capital social accumulé par certains artistes à travers leur socialisation antérieure et/ou leur participation à d'autres scènes sociales peut alors constituer une ressource décisive pour intégrer ces réseaux de diffusion. La compagnie de Dominique obtient par exemple son premier engagement (un spectacle pour enfants) par l'intermédiaire de la MJC où il est animateur; le réseau militant d'un autre membre de la compagnie leur permet aussi de se produire à l'occasion de rassemblements.

Après leurs débuts, les artistes de cette génération ont fait preuve d'une importante longévité professionnelle. La plupart ont travaillé plus de vingt ans en marge du réseau de diffusion subventionné pour ensuite s'éloigner de la scène. Dans un contexte de concurrence limitée du fait d'effectifs encore restreints, leur carrière s'est souvent déroulée uniquement au sein des compagnies fondées initialement. Affinités électives et professionnelles sont ainsi mêlées, sur le modèle idéal-typique de la troupe ou de la communauté artistique (Proust, 2003). Manifestant une certaine distance vis-à-vis des institutions (scolaire notamment) et valorisant la vie de bohème, plusieurs interviewés expriment aussi une vision critique des administrations culturelles et de leur fonctionnement:

Le rapport aux institutions qui, forcément, devient politique, stratégique, tout ça, ça ne me plaisait pas du tout. [...] Oui, tenir un discours artistique alors que je n'arrivais même pas à m'assumer en tant qu'artiste, je me demandais toujours ce qu'ils voulaient que je leur dise, je n'avais pas de discours, je faisais quelque chose et puis j'avais envie que les gens programment ça ou non. Voilà, c'est tout. Mais dans le milieu culturel, ça ne suffit pas. (Xavier, né en 1967, père enseignant de philosophie, mère sage-femme)

Le parcours autodidacte de Xavier, devenu acrobate aérien après une pratique intensive de l'escalade puis de la danse, ainsi que ses faibles ressources scolaires (arrêt du lycée à 17 ans) expliquent en partie sa difficulté à répondre aux injonctions institutionnelles de production d'un discours réflexif sur les œuvres. Sa réticence à se définir comme un "artiste» est d'ailleurs partagée par d'autres membres de cette génération qui préfèrent se décrire comme des « artisans » du cirque, insistant alors sur les savoir-faire acquis par l'expérience. Cette forme de désignation de soi peut être mise 
en lien avec la dimension informelle et pratique de leur socialisation au métier (Tolley, 2004). À l'inverse, Michel et Patrice, plus anciens et socialisés au théâtre pendant plusieurs années, ont repris à leur compte les notions de « créateurs » ou d'« auteurs » d'œuvres, contribuant ainsi au processus de reconnaissance artistique du cirque. Alors qu'un seul artiste de cette génération s'est formé quelques mois au sein d'une école professionnelle, c'est le cas de tous ceux de la génération suivante, ce qui traduit le caractère désormais central de cette voie d'accès au métier.

\section{Artistes de cirque contemporain : la forme scolaire comme condition d'accès à la profession}

Seize artistes (dont huit femmes) nés entre 1975 et 1981 présentent des dispositions et des modes de socialisation distincts des artistes du nouveau cirque. Leur découverte du cirque s'est faite plus précocement même si elle reste plus tardive que dans d'autres métiers artistiques ou sportifs (Sorignet, 2001 ; Bertrand, 2008). Plus précisément, c'est principalement lors de leurs études secondaires que ces artistes découvrent le cirque, activité de loisirs alors en plein essor en France.

\subsection{Le cirque : une voie d'accès genrée vers l'art}

Parallèlement au développement d'écoles professionnelles, de nombreuses écoles de cirque loisir émergent en France à la fin des années $1980^{\circ}$, ce qui favorise l'intérêt de certaines familles pour cette offre de loisirs. Par exemple, la mère de Gaël (aujourd'hui acrobate et jongleur) propose à son fils de s'inscrire à un cours de cirque lorsqu'il est adolescent: «Elle a découvert qu'il y avait une école de cirque [...]. Elle avait vu que j'avais adoré la colonie [de cirque], elle m'a dit "ça te dirait d'en faire ?" et j'ai dit “oui !" » (Gaël, né en 1975, mère professeure de mathématiques, père explorateur).

Enseignante dans le secondaire, la mère de Gaël joue un rôle prépondérant dans sa socialisation artistique. Les artistes de cette génération sont issus pour la plupart de familles dont l'un des parents exerce une profession intellectuelle supérieure (ingénieur, consultant, professeurs du secondaire ou des écoles, etc.) et, pour plus de la moitié, dans le secteur public. Les travaux conduits par Christine Mennesson (2011) ont montré que les classes moyennes et supérieures dotées en capital culturel tendent à valoriser, parmi l'offre de loisirs, la pratique artistique pour ses vertus épanouissantes plutôt que le sport de compétition, davantage mis à distance. Cette catégorie de la population se tourne en outre de façon privilégiée vers des pratiques sociales en cours de légitimation (Bourdieu, 1979) dont le cirque fait alors partie. Avant de choisir cette

\footnotetext{
8 En 2016, la Fédération française des écoles de cirque (FFEC), créée en 1988, comptait
} 143 écoles de cirque amateurs adhérentes en France. 
activité artistique, les hommes de cette génération ont d'ailleurs expérimenté plusieurs loisirs sportifs :

Ouais, moi j'ai tout fait, j'ai changé de sport tous les ans, donc... Là [le cirque], c'est le seul sur lequel je suis resté... [...] J'ai fait de la voile, de l'équitation, du baseball, du foot... [...] II y avait une école de cirque, je me suis dit: "Tiens chouette, ça me changera du foot !" ». (Antoine, né en 1978, jongleur, père directeur commercial, mère gérante d'un magasin)

En retraçant son parcours en matière de loisirs, Antoine évoque des activités qui suggèrent un goût pour des pratiques émergentes dans les années 1990 en France (baseball, cirque). Les membres des classes dotées en capital culturel tendent aussi à privilégier la diversité et la modération dans leur choix en matière d'activités sportives (Mennesson, 2011). En école amateur, le cirque réunit ces deux traits. Il apparaît d'abord comme un moyen de prolonger chez les artistes hommes de cette génération une socialisation corporelle variée puisque les écoles de loisir forment aux différents " arts " du cirque : jonglerie, équilibre, aériens (trapèze, corde lisse), acrobatie au sol, etc.

Parmi les artistes de cirque contemporain, la socialisation corporelle distingue nettement hommes et femmes. Chez ces dernières, les loisirs antérieurs au cirque se caractérisent par la prégnance de la pratique gymnique, pratiquée dans la durée. En France, les écoles professionnelles ont largement emprunté aux techniques gymniques (acrobatie, acrosport, trampoline) afin d'élever le niveau de performance des artistes9. Les savoir-faire acrobatiques apparaissent ainsi incontournables pour y entrer. Or, ces prérequis tendent à pénaliser celles qui se seraient uniquement formées en écoles amateur. En effet, la forte sexuation des techniques de cirque cantonne souvent les jeunes filles dans des disciplines aériennes fixes (trapèze, tissu) ou de souplesse (contorsion par exemple). Dans ce contexte, la socialisation gymnique, d'autant plus lorsqu'elle s'opère selon une modalité prolongée et compétitive, offre de meilleures chances aux candidates d'intégrer une formation professionnelle. Alors que les hommes ont surtout pratiqué des activités sportives multiples et non compétitives, ce type de socialisation corporelle repéré chez les femmes interrogées est l'occasion d'intérioriser des habitudes de travail permettant d'endurer la sollicitation quotidienne du corps lors de l'apprentissage puis lors de l'exercice du métier :

Tu vois, je faisais de la gym tous les soirs, donc je faisais les devoirs entre midi et deux. Je rentrais chez moi pour faire les devoirs. [...] J'ai fait des compétitions pendant une dizaine d'années. Au lycée, je faisais 12 heures de gym par semaine, j'en faisais pas mal. Du coup, j'avais l'habitude de travailler mon corps,

\footnotetext{
${ }^{9}$ Le faible niveau de prouesse physique constituait d'ailleurs une critique récurrente de la part des professionnels du cirque classique au sujet du nouveau cirque, qualifié de «cirque théâtre ». À la suite d'apprentissages physiques et techniques, ce sont les enseignements artistiques (danse, musique, jeu d'acteur, etc.) ainsi que l'individualisation des techniques corporelles qui permettent d'inscrire les prouesses des artistes de cirque contemporain dans le domaine de la création artistique. Pour plus de détails, voir Salaméro (2009).
} 
on peut dire. (Camille, née en 1981, acrobate, père receveur à la poste, mère professeure de technologie)

À l'instar des «petits rats de l'opéra » (Laillier, 2011), Camille organise son rythme quotidien (devoirs faits entre midi et deux heures, absence de sociabilité avec ses camarades d'école lors de la pause méridienne) pour pouvoir suivre ses nombreux cours de gymnastique hebdomadaires en soirée. Cette intensité ainsi que les nombreuses années de pratique développent chez elle une discipline de travail (scolaire et gymnique), ce que ne permettent pas toujours les écoles de cirque loisirs. En effet, dans la lignée de l'éducation populaire, celles-ci insistent sur la découverte des disciplines de cirque et la familiarisation avec la « création » artistique (élaboration de spectacles ou de numéros joués en public) à des fins de « développement personnel $»^{10}$ (Fédération française des écoles de cirque, 2017). Les valeurs de respect, d'entraide, d'égalité ou encore la valorisation de la singularité, centrales dans l'éthos du cirque contemporain (Garcia, 2011), sont aussi constitutives de la pédagogie prônée en écoles amateurs, notamment celles encadrées par la FFEC. Elles apparaissent congruentes visà-vis des valeurs parentales de cette génération d'artistes et garantissent ainsi leur adhésion à l'activité de loisir.

Au-delà de la dimension corporelle du cirque, ce sont aussi les expériences artistiques qui expliquent le goût pour cette activité. Nombreux sont les artistes de cette génération, plus particulièrement les hommes, à s'être initiés aux spectacles à travers le cirque, comme l'exprime Mathieu :

Je ne connaissais rien en spectacle [avant le cirque]. Non, mais vraiment rien du tout. Le spectacle vivant, je n'avais aucune culture: en théâtre, en danse, je n'avais jamais vu de spectacles avec mes parents. Jamais. [...] J'ai découvert le cirque et après les spectacles vivants. [...] [En école amateur] J'ai rencontré quand même des gens, [...] [ça a permis] de voir des spectacles, [...] d'aller à la rencontre des écoles de cirque entre autres, de commencer à entrer dans le milieu du cirque. (Mathieu, né en 1979, jongleur, père propriétaire d'un bateau de pêche, mère aide-soignante)

$\mathrm{Si}$, comme les artistes de nouveau cirque, Mathieu n'est pas issu d'une famille amatrice de spectacles vivants, contrairement à ceux-ci, c'est via l'école de cirque loisir et non par l'exercice du métier au contact des pairs qu'il découvre cet univers, ses institutions et ses acteurs. L'apprentissage en école n'exclut pas des formes de socialisation informelle (Tolley, 2004), en particulier lors d'échanges entre élèves et artistes en activité, mais ces temps informels restent orchestrés par l'école. Son rôle dans la socialisation des artistes s'en trouve accentué, notamment grâce à une continuité entre sphère de l'école et pairs. Chez Mathieu, la pratique amateur permet d'acquérir une culture en matière de spectacles, un réseau spécifique et des connaissances sur le paysage de la formation professionnelle. Ces savoirs sont utiles

\footnotetext{
${ }^{10}$ Fédération française des écoles de cirque, Dossier primo adhésion 2017. En ligne, consulté le 30 juillet 2017. URL: http://www.ffec.asso.fr/download.php?file=3-adherer/2017/dossier_primo_adhesion_2017.pdf.
} 
pour son orientation professionnelle, lorsque vient le moment de choisir une école de cirque. Les artistes de cette génération sont toutefois plus nombreux que ceux de la précédente à avoir connu des expériences scéniques et participé avant leur découverte du cirque à l'élaboration de spectacles de théâtre, de musique ou de danse. C'est davantage le cas des femmes artistes rencontrées qui voient alors dans le cirque une activité voire un métier idéal : «Et je suis tombée sur une pub pour l'ENSAC [...]. C'était tellement mon parcours jusque-là que je me suis dit : "si c'est pas fait pour moi, en tout cas il faut que j’aille voir !" » (Marion, née en 1978, trapéziste, père cadre à la poste, mère au foyer). Ainsi, pour celles qui souhaitent devenir artistes de cirque, les dispositions ascétiques et artistiques apparaissent plus centrales que pour les hommes. Cela laisse penser qu'elles subissent, comme en danse (Sorignet, 2004), une sursélection vis-à-vis de leurs homologues masculins.

Au final, pour la plupart des artistes de cette génération, la pratique du cirque intervient comme une matrice socialisatrice de transition entre le sport et l'art, transition qui s'opérait plutôt entre le milieu de l'animation socioculturelle et le spectacle chez les artistes de nouveau cirque. La fréquentation d'une école de cirque amateur et/ou la pratique antérieure d'autres arts permettent aux artistes de cirque contemporain d'intérioriser de façon plus précoce que leurs pairs de la première génération les critères de la création contemporaine : authenticité, singularité, individualité et valorisation du processus de recherche dans l'élaboration de l'œuvre (Mauger, 2006). En effet, ces valeurs sont centrales dans l'identification professionnelle future au monde de l'art.

\subsection{Un apprentissage scolaire alliant spécialisation et singularisation}

En situation de réussite scolaire relative (résultats moyens, insuffisants pour intégrer des filières sélectives), l'orientation vers le cirque peut apparaître pour les artistes de cirque contemporain comme une manière d'éviter une situation de déclassement social. Contrairement à ceux de la première génération qui ont souvent quitté l'école avant la fin des études secondaires, les artistes de cirque contemporain ont pour une large majorité d'entre eux obtenu leur baccalauréat. Ce dernier est considéré par leurs parents comme un seuil minimal à atteindre. Une minorité (cinq) a même suivi quelques années d'études supérieures (langues, psychologie, STAPS, physique, etc.) avant d'intégrer le cursus d'apprentissage pour le métier d'artiste de cirque. Les artistes de cette génération que nous avons rencontrés ont ainsi appris leur métier en écoles professionnelles, devenues aujourd'hui incontournables pour intégrer le marché du travail artistique le plus légitime (spectacles « de création » subventionnés).

Si le choix de vie des artistes de nouveau cirque a pu faire l'objet de vives réactions parentales, les familles des artistes de cirque contemporain ont soutenu moralement et financièrement leur projet professionnel, malgré leurs inquiétudes liées aux possibilités d'en vivre et leur méconnaissance du métier. Jérôme, acrobate et directeur 
de compagnie, évoque ainsi sa mère, sociologue, «hyper fière » de le voir jouer sur scène. Ce soutien est d'autant plus important que les parcours de formation suivis sont relativement longs (de trois à sept années). Dans les écoles professionnelles, cette durée est pensée comme nécessaire à l'acquisition d'un niveau technique élevé, allant de pair avec une forte spécialisation disciplinaire. Mis à part deux jongleurs, ces professionnels ont choisi les spécialités suivantes : acrobatie au sol et voltige (cinq), portés acrobatiques (quatre), aériens ballant (deux), mât chinois (deux). Si ces interviewés mobilisent plus facilement la figure de l'artiste pour se présenter, ils mentionnent également la discipline pratiquée : «Je dis que je suis artiste de cirque et que je suis spécialisée en bicyclette artistique » (Karina, née en 1977, acrobate, père ingénieur, mère conseillère en assurance).

Formés plus longuement au cirque, qui plus est après des études secondaires, voire supérieures, les artistes de cirque contemporain sont globalement plus diplômés que leurs aînés. Les dispositions scolaires acquises au cours de leur cursus de formation facilitent la maîtrise de compétences administratives (Proust, 2014), de présentation de soi et de compréhension des attentes de diverses instances d'évaluation. Ces dispositions sont en effet nécessaires pour remplir les dossiers d'inscription aux concours d'entrée en écoles professionnelles comme pour l'obtention des subventions publiques, devenues un mode de financement majeur de la création artistique française. À travers la multiformation en écoles, ces artistes apprennent également à évoluer dans des structures dont les modes de fonctionnement peuvent apparaître contraires à leurs propres valeurs. Maxime, acrobate et régisseur dans une compagnie de cirque conventionnée, revient sur son arrivée dans une école de cirque de premier plan. « Voilà, je suis arrivé à Y [école supérieure], il y a M. X. [le directeur] qui fait un beau discours en disant : "Vous allez devenir l'élite du cirque français", chose à laquelle je ne voulais vraiment pas adhérer, j'étais complètement opposé à ça » (Maxime, né en 1977, père informaticien, mère professeure des écoles). À l'entrée en formation, Maxime vit un décalage important entre le discours de type élitiste prononcé dans cette école et ses représentations d'un " cirque pour tous », issues de sa socialisation en école de loisir au cours de laquelle il a pu côtoyer des artistes de nouveau cirque ${ }^{11}$. Une telle conception du cirque entre d'ailleurs en accord avec l'éthos égalitaire des classes moyennes dotées en capital culturel (Mennesson, 2011) dont il est lui-même issu. En dépit de cette tension initiale entre socialisations amateur et professionnelle, visée égalitaire et élitisme - une tension plus marquée pour ceux qui n'ont pas connu de socialisation sportive intense - Maxime est conscient que cette école supérieure lui offrira les meilleures chances de vivre de son art. Il y intériorise le fonctionnement des réseaux les plus légitimes du cirque contemporain, savoir utile pour sa carrière professionnelle en compagnie subventionnée. Ainsi, l'idéal d'un cirque pour tous se heurte rapidement chez les

\footnotetext{
${ }^{11}$ Certains ont été à l'origine d'écoles amateurs ; d'autres interviennent aujourd'hui en écoles professionnelles.
} 
artistes de cirque contemporain aux processus de division du travail et de hiérarchisation des fonctions désormais observables dans les institutions circassiennes professionnelles (Cordier, 2007). Dans ce contexte, leurs aspirations artistiques résultent davantage d'une forme de socialisation professionnelle valorisant réflexivité et expression de soi (Salaméro, Haschar-Noé, 2011). L'individualisation des parcours artistiques est par ailleurs accentuée par la variété de l'offre actuelle de formation professionnelle en matière de cirque en France et en Europe ainsi que par la multiformation. Ce mode d'apprentissage du métier s'accompagne d'une mise à distance du discours anti-institutionnel, voire anti-bourgeois, tenu par les artistes de nouveau cirque ; la valorisation de la singularité tend désormais à faire passer au second plan l'idéal de démocratisation culturelle ${ }^{12}$.

\section{Conclusion}

En mettant en lien l'analyse des parcours d'artistes de cirque avec les processus d'institutionnalisation et de professionnalisation de ce domaine culturel, ce travail apporte une lecture originale des différentes manières d'envisager et de pratiquer ce métier. Comme pour d'autres arts dont la légitimation est récente (Faure, 2004 ; Coulangeon, 1999), la professionnalisation du cirque s'est notamment traduite par une transformation des modes d'accès au métier et donc des profils recrutés, ainsi que des modalités de formation. La socialisation familiale des membres du cirque classique a laissé place à un apprentissage informel entre pairs chez les pionniers du nouveau cirque puis à une scolarisation de l'apprentissage professionnel chez les plus jeunes. Celle-ci a contribué à élever les droits d'entrée et à modifier le recrutement social du groupe professionnel, en favorisant les aspirants ayant acquis au préalable des dispositions et compétences avant tout sportives, mais aussi scolaires et éventuellement artistiques. Cette évolution des modalités de socialisation professionnelle a également entraîné une modification des rapports au métier, qui se traduit par l'existence de figures professionnelles spécifiques. D'un côté, les artistes de nouveau cirque, peu diplômés, polyvalents et plutôt issus de milieux modestes, mettent leurs savoir-faire acquis par l'expérience au service d'un spectacle à visée populaire. De l'autre, les artistes contemporains issus des franges dotées en capital culturel des classes moyennes et supérieures, et spécialisés dans une discipline circassienne, sont préparés à naviguer dans un paysage de plus en plus institutionnalisé (écoles, administrations publiques, programmateurs artistiques, etc.).

Le modèle de l'artiste de cirque « créateur », promu par le ministère de la Culture et par certaines écoles supérieures, n'exclut pas pour autant l'existence d'autres figures, investies par des artistes de cirque évoluant dans des espaces moins légitimes (Garcia,

\footnotetext{
${ }^{12}$ De même chez les metteurs en scène, la transformation des modes d'accès au métier s'est traduite par un affaiblissement des socialisations militantes et de la référence à l'action culturelle, au profit de la reconnaissance par les institutions (Glas, 2015).
} 
2011). Ainsi, au-delà des effets générationnels, la profession compte aujourd'hui différents segments professionnels (Strauss, 1992 ; Octobre, 1999), qui entretiennent des rapports distincts aux publics et aux administrations culturelles. En effet, les spécificités de chaque figure tendent à orienter les artistes qui l'incarnent vers un segment professionnel donné : marché subventionné, évènementiel, festivals de rue, etc. De plus, si la scolarisation du cirque a engendré une homogénéisation des modes d'accès au métier, elle n'empêche pas une certaine diversité des parcours au sein d'un espace de formation hiérarchisé, mais aussi polarisé. C’est cette dernière caractéristique qui permet plus particulièrement de répondre à l'exigence de singularisation centrale dans l'identification au domaine de l'art contemporain. Enfin, comme dans d'autres disciplines artistiques (Ravet, 2003), la féminisation de la profession prend appui sur la scolarisation de l'apprentissage du cirque, processus qui a permis à des femmes dotées en capital sportif d'intégrer un univers traditionnellement masculin.

\section{Bibliographie}

BARRe-MeInZER S. (2004), Le cirque classique, un spectacle actuel, Paris, L'Harmattan ( Arts, esthétique, vie culturelle »).

BERTRAND J. (2008), «Se préparer au métier de footballeur : analyse d'une socialisation professionnelle », STAPS, $\mathrm{n}^{\circ}$ 82, p. 29-41.

Boltanski L. (1975), "La constitution du champ de la bande dessinée », Actes de la recherche en sciences sociales, vol. $1, \mathrm{n}^{\circ} 1, \mathrm{p} .37-59$.

Bourdieu P. (1979), La distinction. Critique sociale du jugement, Paris, Éditions de Minuit (« Le sens commun »).

BOURDIEU P. (1998 [1992]), Les règles de l'art. Genèse et structure du champ littéraire, Paris, Seuil (« Essais »).

BRANDL E. (2006), «Ce que l'institutionnalisation fait à la sociologie des arts et de la culture. L'exemple des musiques amplifiées en région », in S. GIREL (dir.), Sociologie des arts et de la culture. Un état de la recherche, Paris, L’Harmattan ("Arts, esthétique, vie culturelle, sociologie »), p. 333-360.

CORDIER M. (2007), "Le cirque contemporain entre rationalisation et quête d'autonomie », Sociétés contemporaines, $\mathrm{n}^{\circ}$ 66, p. 37-59.

CORDIER M. (2009), Le cirque sur la piste de l'art. La création entre politiques et marchés, thèse de doctorat en sociologie, Nanterre, Université Paris Ouest Nanterre La Défense.

Coulangeon P. (1999), Les musiciens de jazz en France à l'heure de la réhabilitation culturelle, Paris, L’Harmattan (« Logiques sociales - musique et champ social »). 
DAPPORTO E., SAGOT-DuvAUROUX D. (2000), Les arts de la rue, portrait économique d'un secteur en pleine effervescence, Paris, La Documentation Française ("Questions de culture »).

DAVID-Gibert G. (2006), Les Arts du cirque. Logiques et enjeux économiques, Paris, La Documentation française (" Questions de culture »).

DEMAZIERE D. (2004), « Les générations comme catégorie d'analyse », Temporalités, $\mathrm{n}^{\circ} 2$, p. 1-5.

FAURE S. (2004), « Institutionnalisation de la danse Hip-Hop et récits autobiographiques des artistes chorégraphes », Genèses, n 55, p. 84-106.

Demaziere D., SAmuel O. (2010), «Inscrire les parcours individuels dans leurs contextes", Temporalités, $\mathrm{n}^{\circ} 2$. En ligne, consulté le 18 mai 2017. URL: http://journals.openedition.org/temporalites/1167.

FourmauX F. (2006), «Le nouveau cirque ou l'esthétisation du frisson», Ethnologie française, $n^{\circ} 36$, p. 659-668.

FOURNIER P. (2008), « Le travail des générations : confronter des présents décalés » in AM. ARBoRIO et al. (dirs.), Observer le travail. Histoire, ethnographie, approches combinées, Paris, La Découverte (« Recherches »), p. 113-129.

GABER F. (2014), « Les arts de la rue et les publics éloignés de la culture », Vie sociale, $n^{\circ} 5$, p. 69-78.

Garcia M.-C. (2011), Artistes de cirque contemporain, Paris, La Dispute (« Arts et culture »).

GLAS M. (2015), « La transformation du phénomène de consécration artistique dans le champ théâtral français, 1950-1990 ", Sociologie et sociétés, vol. 47, nº 2, p. 261-284.

GuY J.-M. (dir.) (2001), Les arts du cirque en l'an 2000, Paris, Association française d'action artistique ("Chroniques de l'AFAA »).

Hammou K. (2011), «Le jeu incertain des générations. La pérennisation du groupe professionnel des rappeurs dans les industries musicales françaises", Temporalités, $\mathrm{n}^{\circ} 14$. En ligne, consulté le 13 décembre 2017. URL: http://journals.openedition.org/temporalites/1918.

LAILLIER J. (2011), « Des familles face à la vocation. Les ressorts de l'investissement des parents des petits rats de l'Opéra », Sociétés contemporaines, $n^{\circ}$ 82, p. 59-83.

LEBON F. (2013), « Les animateurs entre précarité, militantisme et gestion politique des quartiers », Pensée plurielle, n 32 , p. 61-71.

MAUGER G. (dir.) (2006), L'accès à la vie d'artiste: sélection et consécrations artistiques, Bellecombe-en-Bauges, Éditions du Croquant (« Champ social»). 
Mauger G., Poliak C. (2008), «Marginalité, "bonne volonté culturelle” et "bohème populaire" », Savoir/Agir, n 6, p. 67-76.

MENGER P.-M. (1997), La profession de comédien. Formation, activités et carrières dans la démultiplication de soi, Paris, La Documentation Française.

MENNESSON C. (2011), « Socialisation familiale et investissement des filles et des garçons dans les pratiques culturelles et associatives », Réseaux, n 168-169, p. 87-110.

OCtobre S. (1999), « Profession, segments professionnels et identité. L'évolution des conservateurs de musée », Revue française de sociologie, vol. 40, n² 2, p. 357-383.

Proust S. (2003), « La communauté théâtrale », Revue Française de Sociologie, n 44, p. 93113.

PRoust S. (2014), Intériorisation des dispositions professionnelles et tensions salariales, Rapport de recherche, Lyon, Centre Max Weber.

RAVET H. (2003), « Professionnalisation féminine et féminisation d'une profession : les artistes interprètes de musique », Travail, genre et sociétés, $n^{\circ}$ 9, p. 173-195.

SALAMERO É. (2009), Devenir artiste de cirque: espace des écoles et socialisation professionnelle, thèse de doctorat STAPS, Toulouse, UFR STAPS.

SAlAmERO É., HASCHAR-NOE N. (2011), « Fabriquer un artiste créateur : formes et effets des dispositifs de socialisation à la création dans les écoles professionnelles de cirque », Sociologie de l'art, Opus 17, p. 75-94.

Sinigaglia J. (2013), "Le bonheur comme rétribution du travail artistique. De l'injonction à l'incorporation d'une norme », Sociétés contemporaines, n 91, p. 17-42.

SORIGNET P.-E. (2001), Le métier de danseur contemporain, thèse de sciences sociales, Paris, EHESS.

SORIGNET P.-E. (2004), "Être danseuse contemporaine : une carrière "corps et âme" ", Travail, genre et sociétés, $\mathrm{n}^{\circ} 12$, p. 33-53.

StRAUSS, A. (1992), La trame de la négociation: sociologie qualitative et interactionniste, Paris, L’Harmattan (« Logiques sociales »).

TOLLEY C. (2004), «Formation scolaire ou formation sur le tas chez les bergers de Provence. Différenciation des pratiques et conflit de légitimité?», Sociétés contemporaines, $\mathrm{n}^{\circ}$ 55, p. 115-138.

VinCENT G., LAHIRE B., THIN D. (1994), «Sur l'histoire et la théorie de la forme scolaire », in G. VINCENT (dir.), L'éducation prisonnière de la forme scolaire, Lyon, Presses Universitaires de Lyon, p. 11-48. 